\title{
Vascular Disease Risks: Known and the Unknown?
}

\section{Rao GHR}

Emeritus Professor, University of Minnesota, USA

*Corresponding author: Gundu HR Rao, Emeritus Professor, Laboratory Medicine and Pathology, Director, Thrombosis Research, Lillehei Heart Institute, University of

\section{Mini Review}

Volume 2 Issue 3

Received Date: June 12, 2018

Published Date: June 19, 2018 Minnesota, Minneapolis, MN, USA, Email: gundurao9@gmail.com

\section{Abstract}

Acute vascular events such as heart attacks, pulmonary embolisms, and stroke, are the leading causes of mortality and morbidity worldwide. Framingham heart study (FHS), developed by the National Heart Lung\& Blood Institute (NHLBI), of the National Institutes of Health (NIH,) and the Boston University School of Medicine (BUSM), some 70 years ago, developed information about the modifiable risk factors, which promote the development of vascular diseases. Several studies have demonstrated that robust management of modifiable risk factors, leads to the decline in the deaths, due to vascular diseases. A comprehensive study done in 26 industrialized countries, has demonstrated a significant decline in deaths due to cardiovascular diseases (CVDs). The same study also noted that in spite of this observed decline in the CVDassociated deaths, there was an increase in the diabetes-related deaths. Another ongoing study by the National Health and Nutrition Education Survey (NHANES)of the USA, showed that 52\% of adults with overweight, and $32 \%$ of adults with obesity, had no cardiac risk factors (CRFs), or only one CRF. Recent Scientific Statement by the American Heart Association, on the excess diabetes and heart disease in the South Asians living in the USA, also demonstrates the ethnic differences in the manifestation of specific risk factors, and their role in the precipitation of acute vascular events. These and other such observations, raise the question about the possible contribution of risks that are as yet unknown or less known, related to various ethnicity, demography, diet, physical activity, stress, and lifestyle. In this mini overview, we will touch upon the contribution of some known CRFs, speculate on the as yet unknow risks or relatively less known risks, and express our point of view on this very important topic.

Keywords: Cardiovascular Disease; Alzheimer's Disease; Cardio Metabolic; C - Reactive Protein

Abbrevations: CVDs: Cardiovascular Diseases; NHANES: The National Health And Nutrition Education Survey; CRFs: Cardiac Risk Factors; CAD: Coronary Artery Disease; PESA: Progression Of Early Subclinical Atherosclerosis.

\section{Introduction}

Our understanding of the risks for vascular diseases, is derived mostly from the observations made by the researchers of the now famous, Framingham Heart Study [1-5]. Common modifiable risks for vascular diseases are, 


\section{Open Access Journal of Cardiology}

smoking, elevated lipids (cholesterol, triglycerides), low levels of HDL, high blood pressure, hyperglycemia, excess weight, obesity, and type- 2 diabetes. Even if we look at the global literature, much of the information on this topic is derived largely from studies of Caucasians of European origin [6]. Having said that, we would like to inform the readers, that disproportional burden of metabolic diseases, has been noted in various ethnic groups. Yet there are no guidelines, guidance statements, and treatment protocols for ethnic-specific risks for vascular diseases. In the June issue of Circulation, American Heart Association has a scientific statement, which describes the excess diabetes and heart disease burden in the South Asians living in the USA [7]. Researchers from Epidemiology Unit at Cambridge UK, have noted that African-Americans have higher rates of coronary artery disease (CAD) and stroke, compared with Europeans [6]. Whereas, other ethnic populations like Chinese and Japanese, have higher rates of stroke rather than CAD. Contrary to this observation, Mexican Americans seem to have both stroke and heart disease. Native American Indians also suffer from excess weight, obesity, diabetes, and heart disease. These observed differences in the development of CRFs, in various ethnic groups and contribution of these risk factors, collectively or in increments, to the development of acute vascular events, are not very well explained. In some cases, even when the risks are identified and demonstrated, no interventional strategies have been put in place, to combat the increase in the incidence of metabolic diseases. For instance, both India and China have very high incidence of type- 2 diabetes. Over seventy years of epidemiological research done in India (Mysore cohort studies), has shown that low-birth-weight children, are at a greater risk for developing cardio metabolic risks $[8,9]$. In a country like India, 30 percent of the children born, are low birth weight. That means, over 800,000 children are at-risk for developing metabolic diseases every year in India alone. In view of these observations, epidemiology unit of UK, has established a unit at the Mission Hospital, Mysore, India, to study this phenomenon of fetal origin of adult diseases. This is not a unique public health problem to India. It is a well-recognized problem, in many Asian countries including China. There is enough observational evidence to suggest, that marked differences across racial and ethnic groups exists in terms of development of CRFs, and the prevalence of disproportional disease burden. In view of these observations, we should consider that the ethnicity may very well be an independent risk factor, and therefore, ethnic-specific guidelines, guidance statements, and therapeutic modalities should be developed.
According the noncommunicable risk task force (NCDRisc), since 1980, incidence of obesity has increased by two-fold, and diabetes by four-fold $[10,11]$. According to these experts, no country has reversed this trend, in the increase of these epidemics. Chances of achieving the Millennium Development Goals (MDG), set by the United Nations, of arresting the increase in the incidence of these diseases at 2025 to the level of 2020, is next to nothing. How did we reach this disastrous public health tragedy worldwide? What can be done about it? Who is going to do it? These questions are begging for answers and action plans. It is a well-known fact, that individuals with type-2 diabetes have several-fold higher risk, for developing acute vascular events. Excess weight and obesity, also are known to contribute significantly, to the increase in the development of diabetes and CVD related acute events. Even when we talk about obesity, it is important to note, that not all obesity is the same. Have you heard of "Healthy Obesity"? An observational study done, with over 1.3 million adults, by the Center for Health Research, Portland, Oregon, and the staff ofthe Kaiser Permanente, a health care provider, with overweight or obese individuals, showed that these individuals usually have four cardiac risk factors (CFRs), the prevalence of elevated blood pressure, elevated triglycerides, low HDL, and prediabetes. In an ongoing study, by the National Health and Nutrition Examination Survey (NHANES) from 1999-2004, which included both diabetics and obese individuals, they found that $52 \%$ of the adults with overweight, and $32 \%$ of the obese individuals, had no CRFs or only one CRF $[12,13]$.

We just mentioned in the previous paragraph, that not all obesity is the same, what exactly we mean by that? A bilateral study between the Madras Diabetes Research Foundation (MDRF), Chennai, India, and the University of Minnesota, demonstrated that in spite of the lower BMI in the subjects of Chennai, India, compared to the US adults, the waist-to-hip ratio was significantly higher in men and women from Chennai, India. In view of these findings, World Health Organization (WHO) has come up with special ethnic-specific guidance, for BMI cutoffs for this group. The obesity in this population has been described as central abdominal obesity or visceral obesity. Excess visceral fat is supposed to shed exosomes which create chronic, low grade inflammatory state, which predisposes to insulin resistance, and a pre-thrombotic phenotype [14]. Although this is a well-recognized ethnic-specific metabolic risk in South Asian phenotype, not much attention has been given to define the role, or to find ways to reduce or reverse the accumulation of the visceral fat or prevent it from developing in the first place. The fact that over a billion individuals, have an altered lipid 


\section{Open Access Journal of Cardiology}

metabolism, and a genetically programmed deposition of fat around their bellies, which predisposes them preferentially to increased metabolic diseases, should raise the curiosity of researchers, clinicians, care providers and policy makers. Readers interested in learing more about this differential fat deposition between Asian individuals and Caucasians should refer to a seminal article by Professor Chittaranjan Yajnik [15]. In this study they clearly show how the percent deposition of fat is variable in spite of equal BMI and also demonstrate the limitations of using BMI as a measure of adiposity, across various ethnic populations. The contributions of genes in modulating fat distribution across populations, is worth exploring.

In fact, excess weight, obesity (irrespective of visceral or sub-cutaneous), and type-2 diabetes, havesome common metabolic risks such as, inflammation, oxidative stress, endothelial dysfunction, hardening of the arteries, as well as the presence and progress of sub-clinical atherosclerosis. Having said that, we wonder why more attention has not been given, to develop interventions or therapeutic modalities, for these observed risks? Professor Valentin Fuster and associates, from the Mount Sinai Hospital, New York, in their analysis of the progression of early subclinical atherosclerosis (PESA) study, have demonstrated that $50 \%$ of the asymptomatic adults, had subclinical atherosclerosis, and hardened arteries [16]. Researchers at the Rasmussen Center for the Prevention of Cardiovascular Disease, at the University of Minnesota, under the leadership of Professor Jay Cohn, in their ten-point diagnostic approach, monitor endothelial dysfunction $[17,18]$. Dysfunction of the endothelium, one of the largest organ of the body, is the earliest sign of the existence of vascular disease in progress. Experts claim, that in the absence of subclinical atherosclerosis and endothelial dysfunction, there will be no vascular disease. Yet, no routine tests are done in majority of clinics, for detecting these valuable early signs of vascular alterations. There is a great need, to develop cost-effective tests, for the diagnosis of these alterations of vascular function, in the general population. All of these observed risks are modifiable risks, and in an integrated approach to health care, complimentary therapies could be developed, to address these risks, which are not treated in the standard cardiometabolic care.

Inflammatory response of the blood vessels, involve complex signaling between inflammatory cells (neutrophils, lymphocytes, monocytes, macrophages), endothelial cells (ECs), vascular smooth muscle cells (VSMC), and extracellular matrix components [19].
Persistent increase in cytokines, seem to be associated with vascular dysfunction, and vascular disease such as, subclinical atherosclerosis, endothelial dysfunction, and hypertension [20]. Furthermore, researchers at the Children's National Memorial Hospital at Washington DC, have demonstrated yet another mechanism, by which adipose tissue can induce inflammatory response. Their studies have shown that adipocyte-derived exosomes (micro RNAs) contain mediators, capable of activating end-organ inflammatory and fibrotic signaling pathways [21]. Association between hyperglycemia and inflammation, in the initiation and promotion of vascular complications, is well established [22]. Obesity and oxidative stress, also results in chronic inflammation of the vessels, as demonstrated by elevated $\mathrm{C}$ - reactive protein (CRP). Studies from Minnesota, have demonstrated that in children with excess weight, just 8 weeks of moderate exercise, lowered the inflammatory biomarker [23]. As we have discussed in our earlier articles on this topic, inflammation is associated with all the major metabolic risks, including in the precipitation of acute vascular events. Professor Paul Ridker, Director of theCenter for Cardiovascular Disease Prevention, Brigham and Women's Hospital, Boston, Mass, has demonstrated for the first time that lowering the inflammation by using a monoclonal antibody, independent of cholesterol, reduces cardiovascular risk. In a separate study, researchers from Perelman School of Medicine, at the University of Pennsylvania, in collaboration with National Heart, Lung and Blood Institute, have demonstrated that Ustekinumab, a monoclonal antibody used to treat skin conditions, can also improve aortic inflammation [24].

There is considerable evidence, to suggest an important role for the overproduction of reactive oxygen species (ROS), in the pathogenesis of vascular diseases. These ROS can be released from nicotinamide adenine dinucleotide oxidase, xanthine oxidase, lipoxygenase, mitochondria or the uncoupling of nitric oxide synthetase [25]. The transcription factor Nrf2 (nuclear factor, erythroid-2-related factor-2, Nrf-2) for instance, a master regulator of detoxification, anti-oxidant, antiinflammatory and other cytoprotective mechanisms, is raised by health promoting factors. This transcription factor activates the transcription of over 500 genes (so called survival genes) in the human genome, most of which have cytoprotective functions. The most healthful diets such as Mediterranean and Okinawa are rich in Nrf2 raising nutrients. Recent studies however, have demonstrated that induction of Nrf2 and Ho-1 expression by Protandim (a mixture of five phytochemicals; Ashwagandha, Indian Bacopa, Indian Green Tea, China 


\section{Open Access Journal of Cardiology}

Milk Thistle and China Turmeric) is associated with a reduction in oxidative stress and fibrosis, preservation of the right ventricular (RV) microcirculation and RV function [26]. Studies by the pioneer scientist, professor Joe M McCord and associates on the effect of Protandim on various pathways have shown, significant modulation by Protandim not only of pathways involving antioxidant enzymes, but also those related to Colon Cancer, Cardiovascular disease and Alzheimer's disease [27,28].

\section{Conclusions}

Framingham Heart Study, which was initiated some 70 years ago, by the NHLBI and BUSM, has provided valuable information, about the modifiable risk factors responsible for the development of vascular diseases. It is for the first time, has given the much need evidence, about the risks for vascular diseases, to the preventive cardiologists, and the caregivers, and helped them develop appropriate risk management strategies. Several studies have demonstrated that management of these common risk factors by medication, diet, physical activity or by lifestyle changes reduces significantly the vascular risk for developing acute events. In this overview, we have briefly touched upon other risk factors, which are known, but are as yet not considered seriously in any of the major intervention strategies. We also have briefly discussed the over expression of certain risks, in various ethnic groups, compared with the Caucasians of European origin. Furthermore, we have discussed the differences in fat metabolism and fat accumulation in Asian subjects. Compared to other ethnic groups. We also have discussed some early risks such as inflammation, oxidative stress, subclinical atherosclerosis, vascular alterations such as, hardening of the arteries and endothelial dysfunction and expressed our opinion that these metabolic alterations also fall under the category of modifiable risks. They are not really unknown risks, but are not considered important, in major vascular disease prevention programs.

\section{References}

1. Rao GHR, Kakkar VV (2001) Coronary Artery Disease in South Asians: epidemiology, risk factors and prevention. Jaypee Medical Publishers India.

2. Rao GHR, Thanikachalam S (2005) Coronary Artery Disease: Risk promoters, pathophysiology and prevention. Jaypee Medical Publishers India.

3. Rao GHR (2016) Handbook of Coronary artery Disease. Springer Healthcare, New Delhi, India.
4. Rao GHR, Kakkar VV (2018) Coronary Artery Disease: JP Medical Publishers, New Delhi, India.

5. Framingham Heart Study.

6. Forouhi NG, Sattar N (2006) CVD risk factors and ethnicity-A homogenous relationship? Atherosclerosis 7(1): 11-19.

7. Volgman AS, Palaniappan LS, Aggarwal NT, Gupta M, Khandelwal A, et al. (2018) Atherosclerotic cardiovascular disease: in South Asians in the United States: Epidemiology, Risk factors, and treatments. A Statement from the American Heart Association. Circulation.

8. Rao GHR, Gandhi PG, Sharma V (2014) Clinical complications of type- 2 diabetes mellitus in South Asians and Chinese populations. An Overview. J Diabetes Metab 5: 420.

9. Rao GHR, Bharathi M (2016) Mother and child: first step for prevention of cardio metabolic diseases. The Journal Cardiol Photon 109(2): 179-186.

10. NCD Risk Factor Collaboration (NCD-Ris-C) (2017) Worldwide trends in bod-mass index, underweight, overweight, and obesity from 1975-2016: a pooled analysis of 2416 population-based measurement studies in 128.9 million children, adolescent, and adults. Lancet 390(10113): 2627-2642.

11. NCD Risk Factor Collaboration (NCD-Ris-C) (2016) Worldwide trends in diabetes since 1980: a pooled analysis of 751 population-based studies with 4.4 million participants. Lancet. 387 (10027): 1513-1530.

12. Wildman RP, Munter $\mathrm{P}$, Reynolds K, McGinn AP, Rajpathak S, et al. (2008) The obese without cardiometabolic risk factor clustering and the normal weight with cardiometabolic risk factor clustering: prevalence and correlates of 2 phenotypes among US population (NHANES 199-2004) Arch Intem Med 168 (15): 1617-1624.

13. Nichols GA, Schroeder EB, Karter AG, Gregg EW, Desai J, et al. (2015) Trends in diabetes incidence among 7 million insured adults, 2006-2011: the SUPREME-DM project. Am J Epidemiol 181(1): 32-39.

14. King RJ, Ajjan RA (2017) Vascular risk in obesity: Facts, misconceptions and the unknown. Diab Vasc Dis Res 14 (1): 2-13. 


\section{Open Access Journal of Cardiology}

15. Yajnik C, Yudkin JS (2004) The Y-Y paradox. Lancet 363(9403): 163.

16. Fernanez-Friera, Fuster V, Lopez-Melgar, Oliva B, García-Ruiz JM, et al. (2017) Normal LDL-cholesterol levels are associated with subclinical atherosclerosis in the absence of risk factors. J Am Coll Cardiol 70 (24): 2979-2991.

17. Cohn JN, Hoke L, Whitman W, Sommers PA, Taylor AL, et al. (2003) Screening for early detection of cardiovascular disease in asymptomatic individuals. Am Heart J 146(4): 679-685.

18. Duprez DA, Cohn JN (2008) Identifying early cardiovascular disease to target candidates for treatment. J Clin Hypertens(Greenwich) 10(3): 226231.

19. Madamanchi N, Vendrow A, Runge MS (2005) Oxidative stress and vascular disease. Arterioscler Thromb Vasc Biol 25(1): 29-38.

20. Sprague AH, Khalil RA (2009) Inflammatory cytokines in vascular dysfunction and vascular disease. Biochem Pharmacol 78(6): 539-552.

21. Ferrante SC, Nadler EP, Pillai DK, Hubal MJ, Wang Z, et al. (2014) Adipocyte-derived exosomal miRNAs: A novel mechanism for obesity-related disease. Pediatr Res 77(3): 447-454.
22. Donath MY (2014) Targeting inflammation in the treatment of type- 2 diabetes: time to start. Nature Rev Drug Disc 13: 465-476.

23. Kelly A, Wetzateon RJ, Kaiser DR, Steinberger J, Bank AJ, et al. (2004) Inflammation, insulin and endothelial dysfunction in overweight children and adolescents: The role of exercise. J Pediatr 145 (6): 731-736.

24. Ridker PM, Everett BM, Thuren T, MacFadyen JG, Chang WH, et al. (2017) Anti-inflammatory therapy with Canakinumab for atherosclerotic disease. N Engl J Med 377(12): 1119-1131.

25. Csanyi G, Miller FJ (2014) Oxidative stress in cardiovascular disease. Int J Mol Sci 15(4): 60026008.

26. Boggard HJ, Natarajan R, Henderson R, Long CS, Kraskauskas D, et al. (2009) Chronic pulmonary artery pressure elevation is insufficient to explain right heart failure. Circulation 120(20): 1951-1960.

27. Hybertson BM, Gao B, Bose SK McCord JM (2011) Oxidative stress in health and disease: The therapeutic potential of Nrf2 activation. Mol Aspects of Med 32(4-6): 234-246.

28. McCord JM, Fridovich I (1978) The biology and pathology of oxygen radicals. Ann Int Med 89(1): 122127.

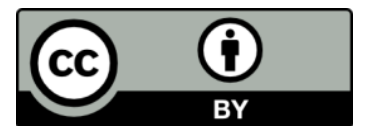

